\title{
Transposon mutagenesis in Mycoplasma hyopneumoniae using a novel mariner-based system for generating random mutations
}

\author{
Gareth A Maglennon ${ }^{1}$, Beth S Cook , Alannah S Deeney ${ }^{1}$, Janine T Bossé ${ }^{2}$, Sarah E Peters ${ }^{4}$, Paul R Langford², \\ Duncan J Maskell ${ }^{4}$, Alexander W Tucker ${ }^{4}$, Brendan W Wren ${ }^{3}$, Andrew N Rycroft ${ }^{1 *}$ and On behalf of the BRaDP1T \\ consortium
}

\begin{abstract}
Mycoplasma hyopneumoniae is the cause of enzootic pneumonia in pigs, a chronic respiratory disease associated with significant economic losses to swine producers worldwide. The molecular pathogenesis of infection is poorly understood due to the lack of genetic tools to allow manipulation of the organism and more generally for the Mycoplasma genus. The objective of this study was to develop a system for generating random transposon insertion mutants in $M$. hyopneumoniae that could prove a powerful tool in enabling the pathogenesis of infection to be unraveled. A novel delivery vector was constructed containing a hyperactive C9 mutant of the Himar1 transposase along with a mini transposon containing the tetracycline resistance cassette, tetM. M. hyopneumoniae strain 232 was electroporated with the construct and tetM-expressing transformants selected on agar containing tetracycline. Individual transformants contained single transposon insertions that were stable upon serial passages in broth medium. The insertion sites of 44 individual transformants were determined and confirmed disruption of several M. hyopneumoniae genes. A large pool of over 10000 mutants was generated that should allow saturation of the M. hyopneumoniae strain 232 genome. This is the first time that transposon mutagenesis has been demonstrated in this important pathogen and could be generally applied for other Mycoplasma species that are intractable to genetic manipulation. The ability to generate random mutant libraries is a powerful tool in the further study of the pathogenesis of this important swine pathogen.
\end{abstract}

\section{Introduction}

Belonging to the class Mollicutes, mycoplasmas are characterised by their lack of a cell wall and small genome size, and are considered to be the smallest free-living self-replicating organisms and as such are of considerable interest in synthetic biology [1]. Respiratory disease is a major problem facing swine producers and Mycoplasma hyopneumoniae, the cause of enzootic pneumonia (EP), is a swine-specific mycoplasma of global prevalence that is one of the leading causes of disease $[2,3]$. EP is characterised by a chronic non-productive cough that is most evident during the growing and fattening stages of production, although all ages of animal may be affected [4].

\footnotetext{
* Correspondence: arycroft@rvc.ac.uk

'Department of Pathology and Pathogen Biology, The Royal Veterinary College, Hawkshead Lane, North Mymms, Hatfield, AL9 7TA, UK Full list of author information is available at the end of the article
}

Mortality rates are usually low, but morbidity may be high with associated economic losses due to increased medication costs, lower growth rates and lower feed conversion efficiencies [2]. The pathogenesis of EP involves entry of $M$. hyopneumoniae into the respiratory tract by inhalation, largely from nose-to-nose contact with other pigs [2], and colonisation of the ciliated epithelial cells of the trachea, bronchi and bronchioles $[5,6]$. Adherence of the organism to the epithelium causes ciliostasis and loss of cilia, thereby preventing effective clearance of debris, pathogens and mucus from the airways [7]. Additionally, M. hyopneumoniae may cause direct cell damage by production of cytotoxic metabolites such as hydrogen peroxide [8]. The chronic nature of infection may result from modulation of the host immune response by M. hyopneumoniae $[2,9]$ and possibly by variable expression of bacterial surface antigens, enabling the organism 
to evade effective clearance [10]. M. hyopneumoniae infection can be associated with and exacerbated by co-infection with other viral pathogens such as Porcine Reproductive and Respiratory Syndrome Virus (PRRSV) and Porcine Circovirus type 2 [2], and with upper respiratory tract bacteria such as Actinobacillus pleuropneumoniae, Streptococcus suis, Haemophilus parasuis and Pasteurella multocida [2]. Commercial $M$. hyopneumoniae vaccines are in widespread use in the pig industry where they are reported to lessen the economic effects of the disease by reducing clinical signs and lung lesions and by improving performance parameters $[3,11,12]$. However, current vaccines are not completely effective and do not prevent colonisation of the respiratory tract with $M$. hyopneumoniae or eliminate infection from the herd [13].

Central to the design of more effective vaccines is an understanding of the pathogenesis of EP. Despite the availability of whole genome sequences of several $M$. hyopneumoniae strains and the small genome size, the functions of most of the protein coding sequences are still unclear. The chronic nature of M. hyopneumoniae infection implies a complicated relationship between the pathogen and the host that is poorly understood. The small genome size of $M$. hyopneumoniae offers an appealing opportunity to understand the molecular basis of disease formation, and to unravel the interplay between the host and the pathogen. Traditionally the exploration of gene function in a bacterium is investigated through the generation of random insertional mutants by transposon mutagenesis. Typically for other Mycoplasmas, the Tn4001 transposon derived from Staphylococcus aureus [14] has been utilised [15-19]. As reviewed by Halbedel and Stülke [20], a mini Tn4001 transposon is used containing an antimicrobial resistance cassette flanked by inverted repeat sequences. The mini transposon and transposase enzyme are delivered into the organism by transformation and expression of the resistance cassette enables selection of mutated organisms. Insertions in essential genes result in lethality but many transformants have mutations in genes that are not necessary for growth in vitro, but may encode for particular pathogenicity determinants that are necessary for growth, survival, invasion or disease in the animal. Such mutants could be exploited in a number of different assays, including in vivo screening of mutants using the powerful technique of signature tagged mutagenesis [21]. Through the screening of large numbers of random mutants in vivo, mutants that are attenuated in vivo can be identified. Such mutants may encode for virulence factors and may be useful in the identification of live attenuated vaccine candidates.

Recently we described the first successful transformation of M. hyopneumoniae strain 232 using an artificial self-replicating oriC plasmid system [22]. This system allowed us to optimise a set of transformation conditions, antimicrobial selection cassettes and promoter sequences for $M$. hyopneumoniae. In this study, the results of that work have been used further to develop a transposon mutagenesis system for M. hyopneumoniae. A key feature of this system is the use of a Himar1 transposon, belonging to the Mariner family of transposons. Insertion occurs at any TA dinucleotide [23] offering an excellent potential for complete coverage of the AT-rich genome of $M$. hyopneumoniae. We describe the development of this system and its use in generating a large pool of several thousand M. hyopneumoniae mutants. This is a major advancement in the study of this important swine pathogen and potentially other Mycoplasmas.

\section{Materials and methods \\ Plasmid construction}

PCR was performed using a Phusion High Fidelity kit (NEB Ltd, Hitchin, UK) according to the manufacturer's instructions. Sequences of oligonucleotides used in the study are shown in Table 1 . To construct pTn4001-RVC1 (Figure 1A), the 2293 base pair (bp) tetM gene of the plasmid pSRT2 [24] was amplified by PCR (primers TetM1 and TetM2) and cloned into the $\mathrm{XbaI}$ and Pst restriction sites of the pMiniTn4001PsPuro plasmid [25]. Mariner transposon plasmids were constructed from the plasmid pMiniHimar1BSC1 (Figure 1B) [26]. To construct pMHWT-2, firstly the 2293 bp tet $M$ gene in pSRT2 [24] was amplified by PCR (primers TetM3 and TetM4) and used to replace the puromycin $\mathrm{N}$-acetyl-transferase (pac) gene of pMiniHimar1BSC1 using the MluI and Sbfl restriction sites (Figure $1 C$ ). pMiniHimar1BSC1 contains the Himar1 transposase gene downstream of the native promoter sequence from the Tn4001 transposase. To produce plasmid pMHWT-1 (Figure 1D), the $1050 \mathrm{bp}$ Himar1 transposase gene minus the Tn4001 promoter sequence was PCR-amplified from pMiniHimar1BSC1 (primers Tpn1 and Tpn2), introducing an XhoI cut site at the 3' end and NdeI and NcoI restriction sites at the 5 ' end. This PCR product was cloned into NdeI and XhoI sites of pMHWT-2, replacing the Himar1 gene and Tn4001 promoter sequence, and introducing a new NcoI cloning site. Next, the 619 bp P97 ciliary adhesin gene promoter sequence was amplified from M. hyopneumoniae strain 232 [27] genomic DNA with NcoI and NdeI restriction sites added (primers P97-1 and P97-2). The P97 promoter sequence was then cloned into the $N c o$ I and NdeI restriction sites upstream of the Himar1 transposase gene, placing Himar1 under its control, and completing plasmid pMHWT-1. To generate plasmid pMHC9-1 (Figure 1E), the 1050 bp hyperactive C9 transposase mutant of Himarl was PCR amplified from plasmid pET29b + C9 [28], adding XhoI and NcoI restriction sites (primers Tpn1 and Tpn2). This PCR product was used to replace the wild-type Himar1 transposase gene 
Table 1 Sequences of oligonucleotides.

\begin{tabular}{lll}
\hline Oligonucleotide & Restriction sites & Oligonucleotide sequence $\mathbf{( 5}^{\prime}$ - $\mathbf{3}^{\prime} \mathbf{)}$ \\
\hline TetM1 & Pstl & GCCGCTGCAGAATTAAAATTAGTG \\
TetM2 & Xbal & GAAATCTAGATTATATAACAACTTAAATTAC \\
TetM3 & Sbfl & CGATCCTGCAGGCAGAATTAAAAGTTAGTG \\
TetM4 & Mlul & CAGTACGCGTTATATAACAACTTAAATTAC \\
Tpn1 & Ndel/Ncol & GCGCATATGCCATGGAAAAAAAGGAATTCGTG \\
Tpn2 & Xhol & CGCCACTCGAGATTATTCAACATAGTTCCCTTC \\
P97-1 & Ndel & CGATCATATGACGGGGATTAAAACAGAAAC \\
P97-2 & Ncol & GAATCCATGGCACCAACAATTCCGGCAGTC \\
TetMF & N/A & GTGGACAAAGGTACAACGAG \\
TetMR & N/A & CGGTAAAGTCGTCACACAC \\
Linker-A & N/A & CGACTGGACCTGGA \\
Linker-B & N/A & GATAAGCAGGGATCGGAACCTCCAGGTCCAGTCG \\
L-PCR-C & N/A & GATAAGCAGGGATCGGAACC \\
L-PCR-L & N/A & GATAGGGTGAGTGTGTC \\
L-PCR-R & N/A & TAGTCGGATAGATAAAGTAC
\end{tabular}

Sequences of oligonucleotides used in the study are shown. Where appropriate, the restriction enzyme recognition sites added to the $5^{\prime}$ end of the oligonucleotides are underlined.

of pMHWT-1, generating plasmid pMHC9-1 [GenBank accession number KF861545] such that the C9 mutant Himar1 is under control of the P97 promoter sequence.

\section{Bacterial culture}

M. hyopneumoniae strain 232 [27] was grown in Friis broth medium at $37{ }^{\circ} \mathrm{C}$ in a static incubator [29]. For growth on solid medium, Friis medium was solidified by addition of
$0.8 \% \mathrm{w} / \mathrm{v}$ purified agar (Oxoid Ltd, Basingstoke, UK) and $0.01 \%$ w/v DEAE-dextran (Sigma-Aldrich Ltd, Gillingham, $\mathrm{UK}$ ) and incubated at $37{ }^{\circ} \mathrm{C}$ with $5 \% \mathrm{CO}_{2}$. For the selection of transformants, tetracycline hydrochloride (Sigma-Aldrich Ltd, Gillingham, UK) was added to Friis broth medium and Friis agar medium at final concentrations of $0.5 \mu \mathrm{g} / \mathrm{mL}$ and $0.2 \mu \mathrm{g} / \mathrm{mL}$ respectively. Molecular cloning was performed using Escherichia coli strain DH5 $\alpha$, grown in Luria-Bertani
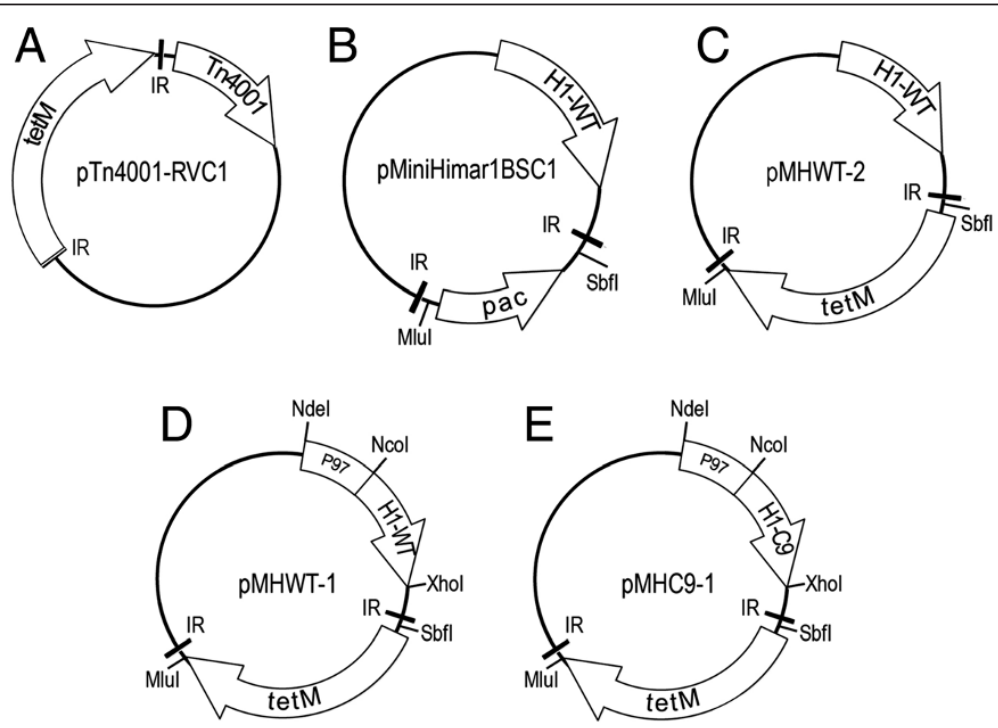

Figure 1 Plasmid maps and construction. Plasmid pTn4001-RVC1 was constructed by replacing the pac gene of pMiniTn4001PsPuro with tetM from plasmid pSRT2 (A). Plasmid pMiniHimar1BSC1 contains a mini transposon incorporating pac conferring resistance to puromycin and the wild-type Himar1 transposase downstream of the Tn4001 native promoter sequence (B) [26]. In the pMHWT-2 plasmid, the pac in pMiniHimar1BSC1 has been replaced with tetM from pSRT2 (C). In plasmid PMHWT-1, the promoter sequence of the M. hyopneumoniae strain 232 P97 gene has been placed upstream of the wild-type Himar1 gene (D). In pMHC9-1, the wild-type Himar1 gene has been replaced with the hyperactive C9 mutant version of the gene (E) [28]. 
medium according to standard methods [30]. Transformants were selected by the addition of $100 \mu \mathrm{g} / \mathrm{mL}$ ampicillin (Sigma-Aldrich Ltd, Gillingham, UK) to the medium. Additionally, for the selection of transformants containing the tet $M$ gene, tetracycline hydrochloride was added to a final concentration of $5 \mu \mathrm{g} / \mathrm{mL}$.

\section{Transformation of mycoplasmas}

M. hyopneumoniae strain 232 was grown to mid-late logarithmic phase in Friis broth as determined by an acid colour change in the phenol red $\mathrm{pH}$ indicator. Mycoplasmas were harvested by centrifugation of culture at $9000 \times \mathrm{g}$ for $10 \mathrm{~min}$ at $4{ }^{\circ} \mathrm{C}$ and washed three times in electroporation buffer (272 $\mathrm{mM}$ sucrose, $8 \mathrm{mM}$ HEPES, $\mathrm{pH}$ 7.4). One hundred microliters of cells (corresponding to $3 \mathrm{~mL}$ culture) were incubated on ice with approximately $10 \mu \mathrm{g}$ plasmid DNA for $30 \mathrm{~min}$. Electroporation was performed in a $0.2 \mathrm{~cm}$ cuvette (Bio-Rad Ltd, Hemel Hempstead, UK) at $2.5 \mathrm{kV}, 100 \Omega, 25 \mu \mathrm{F}$ and $900 \mu \mathrm{L}$ icecold Friis medium were immediately added. After $15 \mathrm{~min}$ incubation on ice, cells were transferred to a $1.5 \mathrm{~mL}$ tube and incubated at $37^{\circ} \mathrm{C}$ for $3 \mathrm{~h}$. Culture was then plated onto Friis agar containing tetracycline and incubated at $37{ }^{\circ} \mathrm{C}$ in $5 \% \mathrm{CO}_{2}$ for up to 18 days. Individual tetracyclineresistant transformants were picked using a sterile pipette tip into Friis broth medium containing tetracycline and grown for 5-7 days until evidence of growth as determined by the $\mathrm{pH}$ indicator.

\section{Determination of transposon insertion sites}

Transposon insertion site determination was based on the method described by Chaudhuri et al. [31]. Genomic DNA was extracted from M. hyopneumoniae strain 232 culture using a phenol-chloroform method [32]. Presence of tet $M$ and plasmid backbone was determined by PCR using primer pairs TetMF/TetMR and Tpn1/Tpn2 respectively. Two and a half micrograms of DNA were digested overnight at $37{ }^{\circ} \mathrm{C}$ with 10 units of $A l u \mathrm{I}$, which cuts the $892 \mathrm{kbp}$ genome of $M$. hyopneumoniae strain 232 a total of 2239 times and generates blunt ends. AluI also cuts within the tet $M$ transposon sequence six times. The digested DNA was purified using a MinElute PCR Purification kit (Qiagen Ltd, Manchester, UK). Oligonucleotide linkers were attached to the digested DNA fragments. To generate the linker, $10 \mu \mathrm{M}$ oligonucleotides Linker-A and Linker-B (Table 1) were heated together in a boiling water bath for $3 \mathrm{~min}$ in annealing buffer (10 mM Tris-HCl, 50 mM NaCl, 1 mM EDTA, pH 8.0) and then allowed to cool for $1 \mathrm{~h}$ at room temperature. Fifty nanograms of AluI digested DNA were bluntligated to $4 \mu \mathrm{L}$ annealed oligonucleotides Linker-A and Linker-B in a $10 \mu \mathrm{L}$ reaction using a Quick Ligation kit (NEB Ltd, Hitchin, UK) for $1 \mathrm{~h}$ at room temperature. The ligated DNA was purified using a MinElute PCR
Purification kit (Qiagen Ltd, Manchester, UK). PCR was performed using HotStarTaq (Qiagen Ltd, Manchester, UK) according to the manufacturer's instructions. Thermocycler conditions were as follows: $95{ }^{\circ} \mathrm{C}$ for $15 \mathrm{~min}$; 33 cycles of $94{ }^{\circ} \mathrm{C}$ for $45 \mathrm{~s}, 55^{\circ} \mathrm{C}$ for $60 \mathrm{~s}, 72{ }^{\circ} \mathrm{C}$ for $120 \mathrm{~s} ; 72{ }^{\circ} \mathrm{C}$ for $10 \mathrm{~min}$. PCR products were visualised by electrophoresis in $1.6 \%$ agarose. Transposon insertion sites were determined by the direct sequencing of PCR products.

\section{Southern analysis}

Southern blotting was performed based on previously described methods [22]. Total DNA was extracted from $20 \mathrm{~mL}$ mycoplasma broth culture using a phenolchloroform method [30,32] and $2.5 \mu \mathrm{g}$ were digested to completion with HindIII. DNA was separated by electrophoresis on $0.9 \%$ agarose, blotted onto Hybond-N + membrane (GE Healthcare Ltd, Little Chalfont, UK) and then fixed to the membrane by exposure to UV light. A digoxigenin (DIG)-labeled probe specific for the tet $M$ gene was generated from PCR-amplified DNA (primers TetMF and TetMR, Table 1) using a DIGHigh Prime DNA Labelling and Detection Starter Kit II (Roche Applied Science Ltd, Burgess Hill, UK). This kit was also used to perform pre-hybridisation and hybridisation in accordance with the manufacturer's instructions. The membrane was autoradiographed at room temperature using CL-XPosure Film (Fisher Scientific Ltd, Loughborough, UK).

\section{Results}

\section{Construction of transposon delivery vectors}

Plasmid pTn4001-RVC1 (Figure 1A) was constructed containing the Tn4001 transposase and a mini Tn4001 transposon consisting of the tet $M$ gene under control of the spiralin gene promoter sequence of Spiroplasma citri bounded by inverted repeats. We previously optimised a set of transformation conditions for M. hyopneumoniae using an oriC-based self-replicating plasmid system, and showed that tet $M$ under control of the spiralin gene promoter region was successfully expressed in $M$. hyopneumoniae strain 232 allowing the selection of transformants on Friis agar plates containing $0.2 \mu \mathrm{g} / \mathrm{mL}$ tetracycline [22]. However, despite three separate attempts at transforming $M$. hyopneumoniae strain 232 with pTn4001-RVC using our optimised conditions and our oriC plasmid pMHO-2 [22] as a positive control for transformation, we obtained no transformants. This suggested that the Tn4001 transposase was not functional in M. hyopneumoniae.

A Himar1 transposon delivery vector (pMiniHimar1BSC1) was recently described that successfully generated transposon insertion mutants in the $M$. gallisepticum $\mathrm{R}_{(\text {low) }}$ strain and a small number of transposon insertions in M. hyopneumoniae strain 232 [26]. pMiniHimar1BSC1 consists of the Himar1 transposase under control of the 
promoter sequence from $\mathrm{Tn} 4001$ and a transposon carrying the pac gene conferring resistance to puromycin (Figure 1B). Small numbers of mutants (less than 10 per transformation) were generated in $M$. hyopneumoniae using this vector, but transformation frequencies were very low and protracted recovery times (several days) were required after transformation prior to selection of transformants on solid medium. We attempted to transform M. hyopneumoniae with pMiniHimar1BSC1 and using a self-replicating oriC plasmid (pMHOpuro) as a positive control for transformation [22]. Approximately $10^{6} \mathrm{CFU}$ were transformed with pMiniHimar1BSC1 and with pMHOpuro. On this occasion, no puromycin resistant transformants were generated with plasmid pMiniHimar1BSC1 while an average of $1.5 \times 10^{-4}$ transformants/CFU were produced by the pMHOpuro positive control. To determine whether tet $M$ selection would increase the yield of transformants, pac was replaced with tetM in plasmid pMHWT-2 (Figure 1C). An alternative hypothesis for the paucity of transformants was that the Tn4001 promoter sequence controlling Himar1 transposase expression was poorly active in $M$. hyopneumoniae hence pMHWT-1 (Figure 1D) was made by replacing the Tn4001 promoter sequence regulating Himar1 with the P97 gene promoter of $M$. hyopneumoniae strain 232 [22]. Plasmid pMHC9-1 (Figure 1E) was constructed by replacing the wild-type Himar1 transposase with the C9 mutant form which exhibits increased activity in vitro [28]. Additionally, as a positive control for transformation, mycoplasmas were transformed with the oriC-based plasmid pMHO-2 (containing tet $M$ ). Approximately $10^{6} \mathrm{CFU}$ were transformed with pMHWT-2, pMHWT-1, pMHC9-1 and pMHO-2 and selection plates examined after incubation for 14 days. Plasmid pMHWT-2 failed to generate any tetracycline resistant colonies compared to an average of $4.6 \times 10^{-5}$ transformants/CFU for the $\mathrm{pMHO}-2$ positive control Table 2 . An average of 123 colonies per transformation were found for pMHC9-1 containing the hyperactive C9 mutant transposase compared to 33 colonies for pMHWT-1 containing the wild-type transposase. Transformation was repeated using pMHWT-1 and

Table 2 Transformation frequencies using Himar1 constructs.

\begin{tabular}{lll}
\hline Plasmid & \multicolumn{2}{c}{$\begin{array}{c}\text { Mean transformation frequency } \\
\text { (transformants/CFU) [SE] }\end{array}$} \\
\cline { 2 - 3 } & Experiment 1 & Experiment 2 \\
\hline pMHWT-2 & 0 & $\mathrm{NT}$ \\
pMHWT-1 & $2.5 \times 10^{-6}\left[1.3 \times 10^{-6}\right]$ & $9.0 \times 10^{-8}\left[2.4 \times 10^{-8}\right]$ \\
pMHC9-1 & $9.3 \times 10^{-6}\left[4.5 \times 10^{-6}\right]$ & $3.0 \times 10^{-7}\left[4.6 \times 10^{-8}\right]$ \\
\hline
\end{tabular}

Mean transformation frequencies (with standard errors) are shown for three separate transformations of $M$. hyopneumoniae strain 232 using plasmid constructs pMHWT-1, pMHWT-2 and pMHC9-1 in two separate experiments. In experiment $1,10^{6} \mathrm{CFU}$ were transformed with each plasmid while in experiment $2,10^{8} \mathrm{CFU}$ were transformed.
pMHC9-1 but with approximately $10^{8} \mathrm{CFU}$ per transformation. Again, more transformants were generated with the hyperactive transposase (33 per transformation) compared to the wild-type transposase (10 per transformation). Transformation of an increased number of cells resulted in a drop in the overall transformation efficiency. However, our experiments suggested that the hyperactive C9 mutant Himar1 transposase could account for a 3-4-fold increase in transformation efficiency compared to the wild-type transposase. Morphologically, tetracycline-resistant colonies grown on Friis agar from transformed cells had a uniform appearance that resembled that of colonies grown from non-transformed cells (Figure 2). However, transformants took approximately 3-5 days longer to reach the same colony size as non-transformed cells. Transformed cells formed discrete and well-separated colonies allowing the isolation of individual colonies. Additionally, colonies were relatively non-adherent to the agar surface and could be easily removed by gentle washing with Friis medium. Importantly, in none of the experiments described did we observe colonies in our "no DNA" control transformations, indicating the absence of pseudo-resistance or spontaneous resistance to tetracycline.

\section{Analysis of transformants}

Transformation of M. hyopneumoniae strain 232 with plasmid pMHC9-1 consistently generated tetracycline resistant colonies on Friis agar containing tetracycline. To confirm that tetracycline resistance was due to transposition into the host chromosome and not, for example, due to maintenance of extrachromosomal plasmid DNA, 15 individual colonies from pMHC9-1 transformed M. hyopneumoniae strain 232 were analysed. Each was transferred into $1 \mathrm{~mL}$ Friis broth medium containing $0.5 \mu \mathrm{g} / \mathrm{mL}$ tetracycline. All transformants

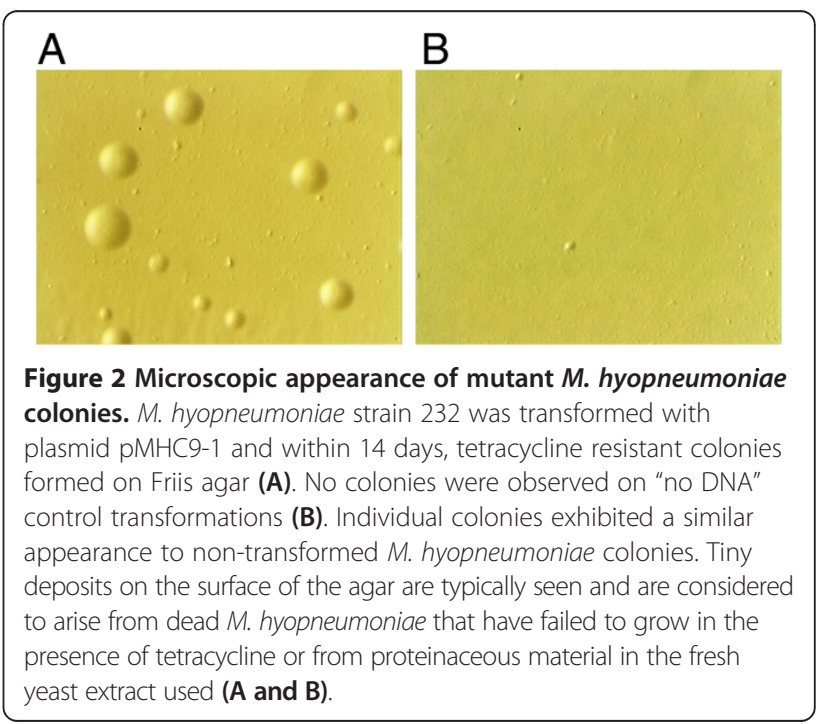


grew within 5 days, confirming the maintenance of tetracycline resistance upon sub-culturing into liquid medium while no growth was observed for untransformed controls. PCR amplification of tet $M$ from total DNA extracts prepared from $20 \mathrm{~mL}$ culture confirmed the presence of tet $M$ in all transformants generated with the hyperactive C9 mutant transposase but not un-transformed M. hyopneumoniae strain 232 (Figure 3A). In addition, PCR for a region of the pUC19 plasmid backbone failed to amplify DNA for any transformant showing that tetracycline resistance was not due to maintenance of extrachromosomal plasmid DNA, or due to integration of the entire plasmid, for example by homologous recombination at the P97 promoter sequence site (Figure 3B).

\section{Analysis of transposon insertion sites}

The presence of tet $M$ and lack of plasmid backbone suggested that transposition of the transposon into the host chromosome had occurred. To locate the site of transposition in randomly selected transformants, a linker PCR technique was employed. Eleven individual tetracycline resistant colonies were colony-purified on Friis agar and cultured in Friis medium containing $0.5 \mathrm{ug} / \mathrm{mL}$ tetracycline. PCR was used to amplify across the site of insertion from the tet $M$ to the linker. This was separately performed on DNA extracts using primer pairs to recognise both ends of the transposon. Analysis showed that a single dominant band was amplified for each transformant using one of the oligonucleotide primer pairs, consistent with integration of the transposon into the host chromosome at one site only. Occasionally, larger feint bands were also present, most likely due to particular PCR parameters such as long extension times and a high number of cycles, or by incomplete digestion of chromosomal DNA by the restriction enzyme AluI. For several transformants, no PCR product was obtained with one of the two oligonucleotide primer pairs, but in all cases, where one primer pair failed, a PCR product was generated with the other primer pair. Therefore, it was possible to confirm independent transposon insertions for all of the 11 transformants. Additionally, the stability of transposon insertion sites following multiple serial passages
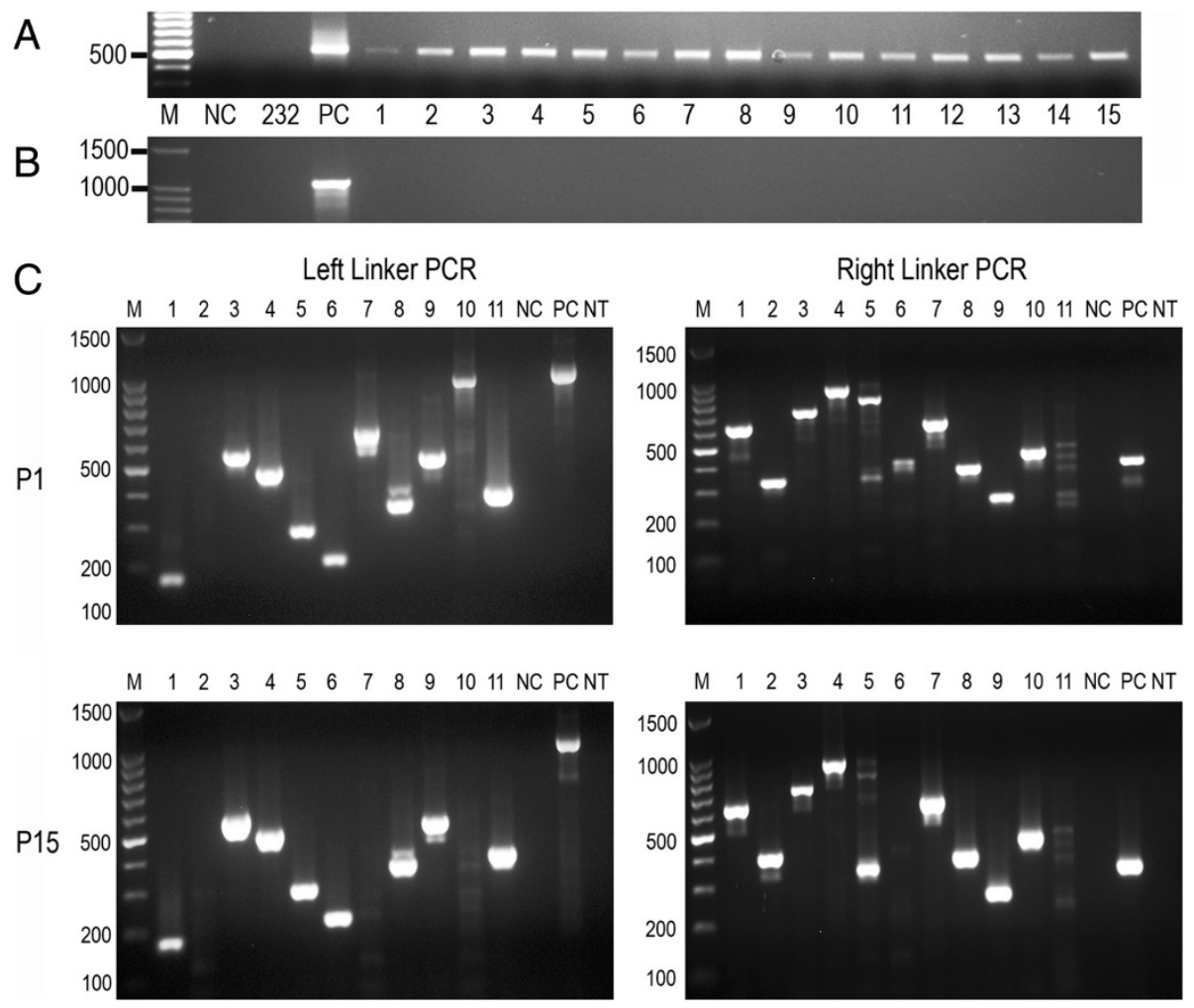

Figure 3 Analysis of mutant $\boldsymbol{M}$. hyopneumoniae by PCR. M. hyopneumoniae strain 232 was transformed with pMHC9-1. PCR was performed on extracted total DNA from 15 individual transformants (lanes 1-15) for identification of the tetM gene (A) and the pGEM-T plasmid backbone (B). A further 11 transformants underwent a further two rounds of "colony purification". Total DNA was extracted and linker PCR performed using two primer pairs at either end of the transposon (left and right) (C). The same 11 transformants were passaged 15 times in Friis medium without selection, and all subsequently retained their resistance to tetracycline. Linker PCR was repeated using the same two primer pairs (left and right). Control samples for PCR reactions included: "no template" control (NT); untransformed M. hyopneumoniae strain 232 (NC); plasmid DNA positive control (PC). 
of individual transformants in non-selective Friis medium was determined. Following a total of 15 passages in Friis medium containing no tetracycline all individual transformants retained their resistance to tetracycline upon subsequent culture in medium containing tetracycline. Linker PCR was repeated for each individual transformant, and agarose gel electrophoresis showed that the amplicons generated were identical in size to those obtained prior to serial passaging. Direct DNA sequencing of PCR amplicons was performed and this confirmed that the transposon had inserted into the host cell chromosome between thymine and adenine residues as expected. Where sequencing at both ends of the transposon was possible, the transposon insertion sites were found to match. Southern analysis was performed on total DNA extracted from the same 11 transformants to confirm that only single transposon insertions were present. For 10 of the mutants, a single band was present using a DNA probe specific for tet $M$ contained within the transposon (Figure 4). For mutant 1, a small intense band was present along with a larger feint band. Only one insertion site was identified for this transformant by linker PCR and this insertion site was confirmed by DNA sequencing. It is possible that two bands were generated by Southern analysis by a failure to adequately digest the DNA to completion with HindIII.

\section{Insertion sites of transposon mutants}

Insertion site sequencing of a further 33 individual transformants was performed so that the locations of a total of 44

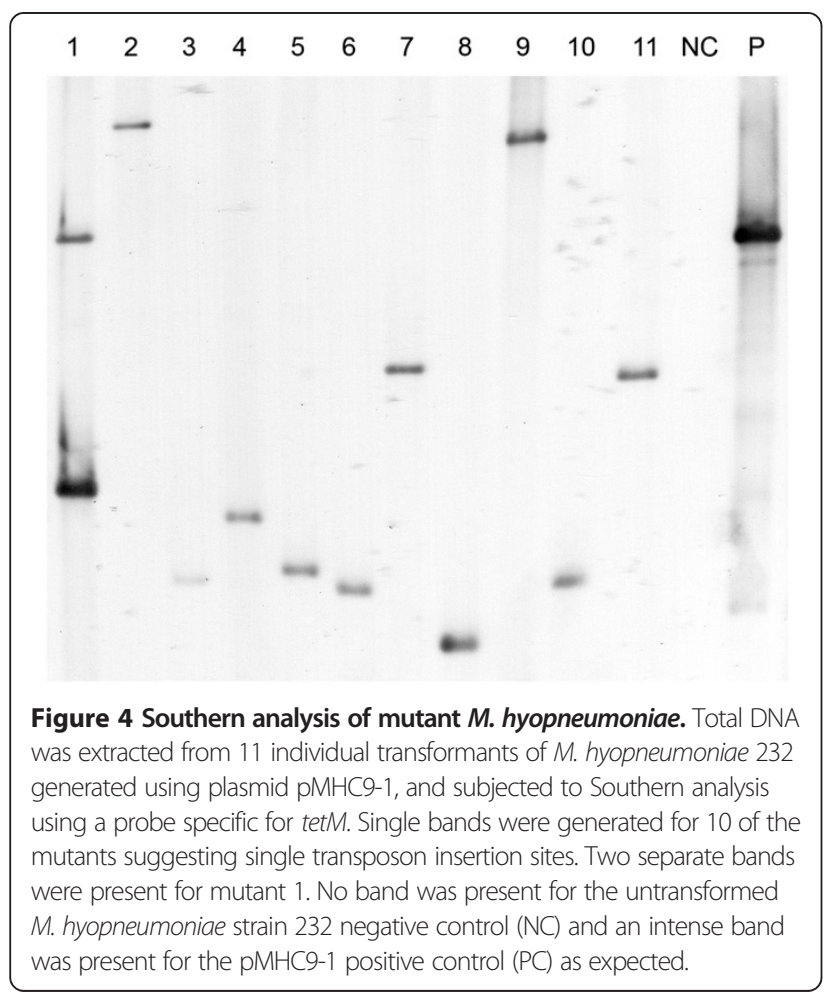

transformants were mapped to the M. hyopneumoniae strain 232 genome (Figure 5). Of these 44 transformants, 41 contained unique insertion sites (Table 3). Mutants 49, 68 and 161 all contained an insertion at the nucleotide 549968 in the M. hyopneumoniae strain 232 genome and mutants 56 and 66 contained identical insertion sites at nucleotide 574428 . It was not possible to determine whether these mutants arose from a single transposition event or from multiple events at the same location. Following electroporation, mycoplasmas are given 3 hours to recover and express tet $M$ in Friis broth without tetracycline prior to plating onto Friis agar containing tetracycline. It is possible that a single transposition event was able to undergo several rounds of multiplication during this period. In total, transposon insertions were present in 22 open reading frames (ORFs) and 5 non-coding regions (NCRs). For 5 ORFs, there was more than one transformant containing an insertion. The average size of an ORF in the M. hyopneumoniae genome is 388 amino acids (aa). All 5 of these ORFs were larger than 388 aa and it is therefore possible that more than one transformant was generated due to the larger size of these ORFs. However, a total of 11 transformants contained a transposon insertion in mhp447. Although this ORF is particularly large at 3970 aa, representing $1.3 \%$ of the $M$. hyopneumoniae strain 232 genome, it is likely that this represents a "hotspot" for transposon insertions. Similarly, 4 transformants contained insertions in the smaller pepF gene (608 aa) and this too may be

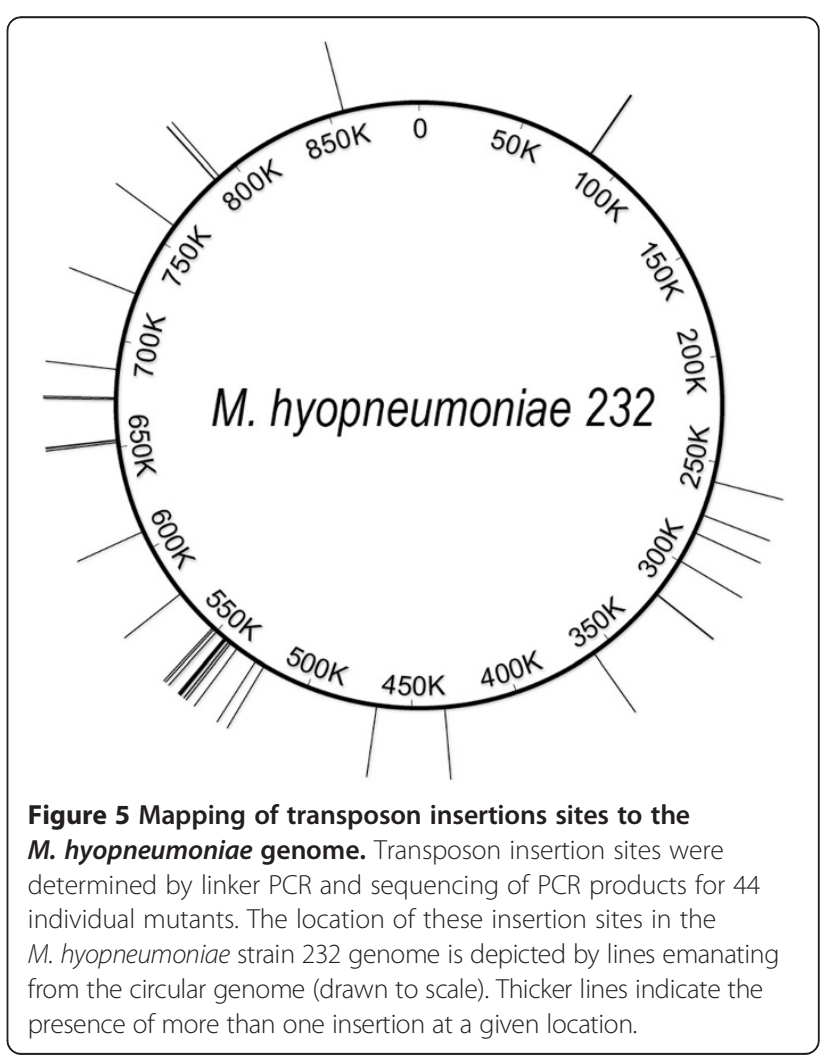


Table 3 Transposon insertion sites of $M$. hyopneumoniae strain 232 mutants.

\begin{tabular}{|c|c|c|c|}
\hline Mutant & Transposon insertion location & ORF disrupted & Gene function \\
\hline 72 & 84920 & Mhp069 & Unknown \\
\hline 11 & 85345 & Mhp069 & Unknown \\
\hline 40 & 259306 & Tig & Trigger factor \\
\hline 159 & 275721 & NCR & Unknown \\
\hline $50 \mathrm{~B}$ & 284811 & Mhp261 & Unknown \\
\hline 4 & 299711 & Mhp271 & P97 ciliary adhesion \\
\hline 96 & 318725 & P95 & Outer membrane protein \\
\hline 165 & 318913 & P95 & Outer membrane protein \\
\hline 91 & 359210 & Mhp313 & Unknown \\
\hline 9 & 434381 & Mhp366 & Unknown \\
\hline 34 & 466299 & ulaA & PTS system ascorbate-specific transporter subunit IIC \\
\hline 37 & 522533 & Mhp443 & Unknown \\
\hline $54 \mathrm{~B}$ & 527019 & Mhp445 & Unknown \\
\hline 156 & 537514 & Mhp446 & Unknown \\
\hline 75 & 540819 & Mhp447 & Unknown \\
\hline $53 \mathrm{~B}$ & 541339 & Mhp447 & Unknown \\
\hline 102 & 542436 & Mhp447 & Unknown \\
\hline 90 & 542541 & Mhp447 & Unknown \\
\hline 1 & 544071 & Mhp447 & Unknown \\
\hline 78 & 544309 & Mhp447 & Unknown \\
\hline 24 & 544767 & Mhp447 & Unknown \\
\hline 5 & 545264 & Mhp447 & Unknown \\
\hline 68 & 549968 & Mhp447 & Unknown \\
\hline 49 & 549968 & Mhp447 & Unknown \\
\hline 161 & 549968 & Mhp447 & Unknown \\
\hline $52 \mathrm{~B}$ & 551617 & NCR & Unknown \\
\hline 13 & 552503 & NCR & Unknown \\
\hline 56 & 574428 & NCR & Unknown \\
\hline 66 & 574428 & NCR & Unknown \\
\hline 17 & 608509 & Mhp490 & PTS system fructose-specific transporter subunit IIABC \\
\hline 16 & 652232 & pepF & Oligoendopeptidase F \\
\hline 162 & 653224 & pepF & Oligoendopeptidase F \\
\hline 101 & 653333 & pepF & Oligoendopeptidase F \\
\hline 92 & 653430 & pepF & Oligoendopeptidase F \\
\hline 47 & 672237 & Mhp535 & Unknown \\
\hline 170 & 672318 & Mhp536 & Unknown \\
\hline 63 & 672979 & Mhp537 & Unknown \\
\hline 50 & 686279 & Mhp542 & Unknown \\
\hline 19 & 722926 & ulaA & PTS system ascorbate-specific transporter subunit IIC \\
\hline 2 & 759509 & NCR & Regulatory region of glpD \\
\hline 10 & 788260 & Mhp630 & Unknown \\
\hline 7 & 788457 & Mhp630 & Unknown \\
\hline 12 & 790862 & Mhp631 & Unknown \\
\hline $51 B$ & 856887 & pepP & xaa-pro aminopeptidase \\
\hline
\end{tabular}


over-represented. Most of the transposon insertions are in genes of unknown function but that appear to be dispensable for axenic growth, or in NCRs. Of the insertions in genes of known function, three were in the P97 ciliary adhesin and the P95 outer membrane protein. Insertions were found in 3 genes encoding phospotransferase system subunit proteins specific for fructose and ascorbate and in the molecular chaperone Trigger Factor. Several insertions were also identified in the pepF gene encoding oligoendopeptidase $\mathrm{F}$ and the pepP gene encoding a proline aminopeptidase. These are two of a small number of proteases that may be associated with processing of M. hyopneumoniae cell surface proteins [33].

\section{Generation of a large pool of $M$. hyopneumoniae mutants}

We sought to determine whether our pMHC9-1 transposon delivery vector could be used to produce a large pool of random mutants, sufficient to provide an adequate number of "hits" in all of the non-essential genes of $M$. hyopneumoniae. A pool of at least 10000 individual mutants would be expected to give a very high probability of an insertion into every one of the 692 protein coding sequences in the M. hyopneumoniae 232 genome determined by Minion et al. [27]. It was calculated that a pool of 10000 individuals would, on average, contain a mycoplasma cell with an insertion every $89 \mathrm{bp}$, and that there would be 14.45 insertions per coding sequence. From the Poisson distribution, 10000 independent insertions would give a 99.99995\% probability of an insertion into each one of the 692 coding sequences. 20 individual transformations were performed using plasmid pMHC9-1 with approximately $10^{6} \mathrm{CFU}$ per transformation. After 14 days growth at $37{ }^{\circ} \mathrm{C}$ on agar plates containing tetracycline, colonies were counted and harvested together in one large pool. A total of 11759 colonies were counted across all 20 plates, representing an average transformation frequency of $3.4 \times 10^{-4}$ transformants/CFU (standard error $5.3 \times 10^{-5}$ ).

\section{Discussion}

The inability to efficiently genetically manipulate $M$. hyopneumoniae has stood as a hurdle to advancements in the understanding of the pathogenesis of enzootic pneumonia in pigs. We recently reported the successful transformation of $M$. hyopneumoniae using an artificial plasmid system containing the origin of replication of M. hyopneumoniae and an antimicrobial resistance cassette, that was capable of self-replicating and maintenance in transformed bacteria [22]. We have used this system to optimise a number of conditions required for the successful transformation of M. hyopneumoniae by electroporation with plasmid DNA. This knowledge has now enabled the design and construction of a transposon-based system for generating random mutants in M. hyopneumoniae.
The Tn4001 transposon is effective in generating insertional mutations in a number of different Mycoplasma species $[16,18,19,34]$ but even using our optimised transformation conditions, we were unable to demonstrate transposition in M. hyopneumoniae. A Himar1 transposon delivery vector that was shown to be active in $M$. gallisepticum generated very low numbers of transformants in $M$. hyopneumoniae with a very restricted range of unique insertion sites [26]. By using an M. hyopneumoniae strain 232-specific promoter sequence to control the Himar1 expression and switching the wild-type Himar1 transposase for a hyperactive mutant C9 form, the frequency of transposition increased significantly. Analysis of transformants confirmed that resistance to tetracycline was due to a transposition event into the mycoplasma genome and it appeared that transposition occurred at a single site in each individual transformant. The transposon insertions appeared to be stable over serial passages and were maintained even in the absence of antimicrobial selection. These features are desirable when the phenotypes of mutants are studied in vitro, and particularly for in vivo studies of pools of mutants by TraDIS or signature-tagged mutagenesis. Additionally, we did not encounter any "pseudoresistant" colonies in our "no DNA" control transformations, which have been documented in transposon mutagenesis systems described for other mycoplasmas $[16,26]$. "Pseudoresistant" colonies can complicate the isolation of true transformants.

In the course of this study, it was evident that there were significant differences in the transformation frequencies obtained using plasmid pMHC9-1. We suspect that differences in transformation frequency can be partially accounted for by variations in the number of cells electroporated and/or the growth phase of the mycoplasma culture used. It appeared that an increase in the number of cells transformed did not necessarily result in an increase in transformation frequency. However, even where similar numbers of cells were electroporated, there could be significant differences in transformation frequencies, implying that other factors could be important. Growth of M. hyopneumoniae is assessed and monitored by a simple change in the phenol red $\mathrm{pH}$ indicator incorporated into the medium, owing to the fact that turbidity is not generated. Thus $\mathrm{pH}$ can serve as a rough guide for growth phase, but the actual number of cells can only be estimated after plating the culture out and allowing growth on agar medium for 5-7 days. Additionally, complex and undefined medium is used to grow M. hyopneumoniae, with $20 \%$ equine/porcine serum and freshly prepared yeast extract contributing to variations in batches of medium.

Transposon insertion sites were determined for a limited number of individual transformants using a linker PCR technique and direct sequencing of PCR products by 
Sanger sequencing. From our limited analysis, we found transposon insertions in a number of $M$. hyopneumoniae genes of both known and unknown function, and in noncoding regions. We observed a clustering of transposon insertions in mhp447, a large open-reading frame of unknown function. The Himar1 transposon inserts at 5'-TA-3' dinucleotides and we considered that the large number of insertions may be due to an increase in AT content. However, analysis of this ORF showed that the AT content was not unusual at 0.681 compared to 0.714 for the entire M. hyopneumoniae strain 232 genome. Alternatively, it is possible that there are hotspots for Himar1 insertions in the M. hyopneumoniae strain 232 genome, that may be affected by factors such as DNA topology, as is the case with other transposons, including Mariner elements [35]. It is difficult to draw any conclusions from analysis of such a small pool of mutants, but determination of the insertion sites of a large pool of mutants by next generation sequencing would answer the question of whether favoured insertion sites do occur. Using plasmid pMHC9-1, we were able to produce a large pool of mutants. Over 10000 colonies were generated through a number of transformations that, given an even distribution should be expected to have a high probability of insertions into all of the genes of $M$. hyopneumoniae. This pool of mutants may prove a valuable tool in future studies of $M$. hyopneumoniae in allowing a list of minimal essential genes to be determined. Such a pool of mutants could also be subjected to an in vivo screen for attenuated mutants using powerful functional genomic techniques such as TraDIS.

A novel transposon delivery system has been generated for M. hyopneumoniae, allowing for the first time the production of large numbers of random transposon insertion mutants. This system has been used to generate mutants with random transposon insertions in individual M. hyopneumoniae protein coding and non-coding sequences. It has also been used to generate a large pool of over 10000 transposon insertions. These mutants are powerful tools in elucidating the functions of $M$. hyopneumoniae genes, and in turn unraveling the pathogenesis of this important swine pathogen.

\section{Additional file}

Additional file 1: BRaDP1T consortium. List of researchers collaborating in the BRaDP1T consortium.

\section{Competing interests}

A patent application covering the method of transposon mutagenesis described in this paper has been filed by the Royal Veterinary College. The authors declare that they have no further competing interests.

\section{Authors' contributions}

Experiments were conceived by GAM, ANR and BSC. Experiments were performed by GAM and ASD. BSC, JTB, ANR, SEP, PRL, BWW, AWT and DJM assisted with experimental design. The manuscript was written by GAM and ANR and was approved by all authors.

\section{Acknowledgements}

This work was supported by a Longer and Larger (LoLa) grant from the Biotechnology and Biological Sciences Research Council (grant numbers BB/G020744/1, BB/G019177/1, BB/G019274/1 and BB/G018553/1), the UK Department for Environment, Food and Rural Affairs and Zoetis awarded to the Bacterial Respiratory Diseases of Pigs-1 Technology (BRaDP1T) consortium Additional file 1. The funders had no role in study design, data collection and analysis, decision to publish, or preparation of the manuscript. The BRaDP1T Consortium comprises: Duncan J. Maskell, Alexander W. (Dan) Tucker, Sarah E. Peters, Lucy A. Weinert, Jinhong (Tracy) Wang, Shi-Lu Luan, Roy R. Chaudhuri (University of Cambridge); Andrew N. Rycroft, Gareth A. Maglennon, Dominic Matthews (Royal Veterinary College); Brendan W. Wren, Jon Cuccui, Vanessa Terra (London School of Hygiene and Tropical Medicine); and Paul R. Langford, Janine T. Bossé, Yanwen Li (Imperial College London). We thank David Lampe for the gift of the pET29b + C9 plasmid from which the hyperactive C9 mutant Himar1 transposase was derived. We thank Joel Renaudin for the gift of the pSRT2 plasmid.

\section{Author details}

'Department of Pathology and Pathogen Biology, The Royal Veterinary College, Hawkshead Lane, North Mymms, Hatfield, AL9 7TA, UK. ${ }^{2}$ Section of Paediatrics, Imperial College London, St Mary's Campus, London, W2 1PG,

UK. ${ }^{3}$ Department of Pathogen Molecular Biology, London School of Hygiene \& Tropical Medicine, Keppel Street, London, WC1E 7HT, UK. ${ }^{4}$ Department of Veterinary Medicine, University of Cambridge, Madingley Road, Cambridge, CB3 OES, UK

Received: 30 October 2013 Accepted: 2 December 2013

Published: 21 December 2013

\section{References}

1. Gibson DG, Glass Jl, Lartigue C, Noskov VN, Chuang RY, Algire MA, Benders GA, Montague MG, Ma L, Moodie MM, Merryman C, Vashee S, Krishnakumar R, Assad-Garcia N, Andrews-Pfannkoch C, Denisova EA, Young L, Qi ZQ, Segall-Shapiro TH, Calvey CH, Parmar PP, Hutchison CA 3rd, Smith HO, Venter JC: Creation of a bacterial cell controlled by a chemically synthesized genome. Science 2010, 329:52-56.

2. Thacker EL, Minion FC, et al: Mycoplasmosis. In Diseases of Swine. $10^{\text {th }}$ edition. Edited by Zimmerman J, Karriker L, Ramirez A, Schwartz K, Stevenson G. New York: Wiley-Blackwell; 2012.

3. Maes D, Segales J, Meyns T, Sibila M, Pieters M, Haesebrouck F: Control of Mycoplasma hyopneumoniae infections in pigs. Vet Microbiol 2008 126:297-309.

4. Simionatto S, Marchioro SB, Maes D, Dellagostin OA: Mycoplasma hyopneumoniae: from disease to vaccine development. Vet Microbiol 2013, 165:234-242

5. Blanchard B, Vena MM, Cavalier A, Le Lannic J, Gouranton J, Kobisch M: Electron microscopic observation of the respiratory tract of SPF piglets inoculated with Mycoplasma hyopneumoniae. Vet Microbiol 1992, 30:329-341

6. Zielinski GC, Ross RF: Adherence of Mycoplasma hyopneumoniae to porcine ciliated respiratory tract cells. Am J Vet Res 1993, 54:1262-1269.

7. DeBey MC, Ross RF: Ciliostasis and loss of cilia induced by Mycoplasma hyopneumoniae in porcine tracheal organ cultures. Infect Immun 1994, 62:5312-5318

8. Razin $S$, Yogev D, Naot Y: Molecular biology and pathogenicity of mycoplasmas. Microbiol Mol Biol Rev 1998, 62:1094-1156.

9. Rodriguez F, Ramirez GA, Sarradell J, Andrada M, Lorenzo H: Immunohistochemical labelling of cytokines in lung lesions of pigs naturally infected with Mycoplasma hyopneumoniae. J Comp Pathol 2004, 130:306-312.

10. Bogema DR, Deutscher AT, Woolley LK, Seymour LM, Raymond BB, Tacchi JL, Padula MP, Dixon NE, Minion FC, Jenkins C, Walker MJ, Djordjevic SP: Characterization of cleavage events in the multifunctional cilium adhesin Mhp684 (P146) reveals a mechanism by which Mycoplasma hyopneumoniae regulates surface topography. MBio 2012, 3:e00282-11.

11. Maes D, Deluyker H, Verdonck M, Castryck F, Miry C, Vrijens B, Verbeke W, Viaene J, de Kruif A: Effect of vaccination against Mycoplasma 
hyopneumoniae in pig herds with an all-in/all-out production system. Vaccine 1999, 17:1024-1034

12. Maes D, Deluyker H, Verdonck M, Castryck F, Miry C, Lein A, Vrijens B, de Kruif A: The effect of vaccination against Mycoplasma hypopneumoniae in pig herds with a continuous production system. Zentralbl Veterinarmed $B$ 1998, 45:495-505.

13. Thacker EL, Thacker BJ, Young TF, Halbur PG: Effect of vaccination on the potentiation of porcine reproductive and respiratory syndrome virus (PRRSV)-induced pneumonia by Mycoplasma hyopneumoniae. Vaccine 2000, 18:1244-1252.

14. Lyon BR, May JW, Skurray RA: Tn4001: a gentamicin and kanamycin resistance transposon in Staphylococcus aureus. Mol Gen Genet 1984 193:554-556.

15. Cao J, Kapke PA, Minion FC: Transformation of Mycoplasma gallisepticum with Tn916, Tn4001, and integrative plasmid vectors. J Bacteriol 1994, 176:4459-4462.

16. Chopra-Dewasthaly R, Zimmermann M, Rosengarten R, Citti C: First steps towards the genetic manipulation of Mycoplasma agalactiae and Mycoplasma bovis using the transposon Tn4001 mod. Int J Med Microbiol 2005, 294:447-453.

17. Dybvig K, French CT, Voelker LL: Construction and use of derivatives of transposon Tn4001 that function in Mycoplasma pulmonis and Mycoplasma arthritidis. J Bacteriol 2000, 182:4343-4347.

18. Hedreyda CT, Lee KK, Krause DC: Transformation of Mycoplasma pneumoniae with Tn4001 by electroporation. Plasmid 1993, 30:170-175.

19. Pour-El I, Adams C, Minion FC: Construction of mini-Tn4001tet and its use in Mycoplasma gallisepticum. Plasmid 2002, 47:129-137.

20. Halbedel S, Stulke J: Tools for the genetic analysis of Mycoplasma. Int J Med Microbiol 2007, 297:37-44

21. Mazurkiewicz P, Tang CM, Boone C, Holden DW: Signature-tagged mutagenesis: barcoding mutants for genome-wide screens. Nat Rev Genet 2006, 7:929-939.

22. Maglennon GA, Cook BS, Matthews D, Langford PR, Maskell DJ, Tucker AW, Wren BW, Rycroft AN, BRaDP1T consortium: Development of a self-replicating plasmid system for Mycoplasma hyopneumoniae. Vet Res 2013, 44:63.

23. Lampe DJ, Churchill ME, Robertson HM: A purified mariner transposase is sufficient to mediate transposition in vitro. EMBO J 1996, 15:5470-5479.

24. Cordova CM, Lartigue C, Sirand-Pugnet P, Renaudin J, Cunha RA, Blanchard A: Identification of the origin of replication of the Mycoplasma pulmonis chromosome and its use in oriC replicative plasmids. J Bacterio/ 2002, 184:5426-5435.

25. Algire MA, Lartigue C, Thomas DW, Assad-Garcia N, Glass Jl, Merryman C: New selectable marker for manipulating the simple genomes of Mycoplasma species. Antimicrob Agents Chemother 2009, 53:4429-4432.

26. Cook B: Development of genetic tools for functional genomic analysis of Mycoplasma hyopneumoniae. University of London, Royal Veterinary College: PhD thesis; 2013

27. Minion FC, Lefkowitz EJ, Madsen ML, Cleary BJ, Swartzell SM, Mahairas GG: The genome sequence of Mycoplasma hyopneumoniae strain 232, the agent of swine mycoplasmosis. J Bacteriol 2004, 186:7123-7133.

28. Lampe DJ, Akerley BJ, Rubin EJ, Mekalanos JJ, Robertson HM: Hyperactive transposase mutants of the Himar1 mariner transposon. Proc Natl Acad Sci USA 1999, 96:11428-11433.

29. Kobisch M, Friis NF: Swine mycoplasmoses. Rev Sci Tech 1996, 15:1569-1605.

30. Sambrook JJ, Russell DDW: Molecular Cloning: A Laboratory Manual. Cold Spring Harbor: Cold Spring Harbor Laboratory Press; 2001

31. Chaudhuri RR, Allen AG, Owen PJ, Shalom G, Stone K, Harrison M, Burgis TA, Lockyer M, Garcia-Lara J, Foster SJ, Pleasance SJ, Peters SE, Maskell DJ, Charles IG: Comprehensive identification of essential Staphylococcus aureus genes using Transposon-Mediated Differential Hybridisation (TMDH). BMC Genomics 2009, 10:291.

32. Bashiruddin JB: Extraction of DNA from mycoplasmas. Methods Mol Biol 1998, 104:141-144

33. Burnett TA, Dinkla K, Rohde M, Chhatwal GS, Uphoff C, Srivastava M, Cordwell SJ, Geary S, Liao X, Minion FC, Walker MJ, Djordjevic SP: P159 is a proteolytically processed, surface adhesin of Mycoplasma hyopneumoniae: defined domains of P159 bind heparin and promote adherence to eukaryote cells. Mol Microbiol 2006, 60:669-686.
34. French $C T$, Lao P, Loraine AE, Matthews BT, Yu H, Dybvig K: Large-scale transposon mutagenesis of Mycoplasma pulmonis. Mol Microbiol 2008, 69:67-76.

35. Claeys Bouuaert C, Chalmers R: Hsmar1 transposition is sensitive to the topology of the transposon donor and the target. PLoS One 2013, 8:e53690.

doi:10.1186/1297-9716-44-124

Cite this article as: Maglennon et al:: Transposon mutagenesis in Mycoplasma hyopneumoniae using a novel mariner-based system for generating random mutations. Veterinary Research 2013 44:124.

\section{Submit your next manuscript to BioMed Central and take full advantage of:}

- Convenient online submission

- Thorough peer review

- No space constraints or color figure charges

- Immediate publication on acceptance

- Inclusion in PubMed, CAS, Scopus and Google Scholar

- Research which is freely available for redistribution

Submit your manuscript at www.biomedcentral.com/submit
Ciomed Central 